\title{
Evolution of Breast Cancer Classification Towards Personalized Medicine
}

\author{
Naila Irum Hadi \\ Professor, Director Research \& Development, Islamabad Medical and Dental College
}

Breast cancer $(\mathrm{BC})$ is a heterogenous disease, both genetically as well as clinically ${ }^{1}$ with need for a histological, molecular and functional classification system that can aid in devising an appropriate treatment and predicting clinical behavior. ${ }^{2}$

"Histological classification" has been considered a valuable tool for classification of breast cancer subtypes for decades owing to its diagnostic as well as limited prognostic value. It characterizes different types on the basis of histological features like glandular/tubular formation, nuclear pleomorphism, and mitotic rate/10 HPF. ${ }^{2}$

Luminal $A$, luminal $B$, basal-like, normal breast like and ErbB2+/Her2+3,4 and recently "claudin-low" 5 have been identified as molecular subtypes of breast cancer on microarray-based gene expression and hierarchical clustering analysis; hence the evolution of "molecular classification" with great potential for predicting therapeutic response and a better ability to predict overall survival as well as disease-free survival and therefore make prognostic predictions. ${ }^{3,4}$

These gene expression patterns have been linked with variations in DNA copy number and mRNA. For example, Estrogen Receptor (ER) positive tumors (luminal $A$ and $B$ ) exhibited PIK3CA (Phosphatidylinositol-4,5-Bisphosphate 3-Kinase Catalytic Subunit Alpha) mutations and ER/PR/HER2 negative presented with P53 mutations. ${ }^{6}$ Breast cancer researchers have developed PAM50 (50gene signature using qRT-PCR), ${ }^{7}$ Oncotype DX (21-gene recurrence score) and MammaPrint (70-gene signature), ${ }^{8}$ of which the latter two have been approved by Food and Drug Administration (FDA) for use in United States. PAM50, now named as Prosigna Breast Cancer

\section{Correspondence:}

Professor Dr. Naila Irum Hadi

Email: naila.irum@imdcollege.edu.pk
Prognostic Gene Signature Assay analyzes genes in early stage, ER-positive BC, while Oncotype DXTM (Genomic Health Inc., Redwood City, CA) gives information regarding recurrence as well as response to chemotherapy in early stage, ER-positive BC. MammaPrint ${ }^{\mathrm{TM}}$ (Agendia, The Netherlands) gene signature, also done in early stage $\mathrm{BC}$ gives information regarding low-/high-risk of development of metastasis, with the option of not administering chemotherapy to patients with low risk hence sparing them the harmful side effects of these drugs.

Cancer stem cells (CSCs), an evolving area of cancer research with likely focus on targeted therapy, provides the "functional classification" for breast cancer based on the hypotheses that breast cancer heterogeneity is either due to oncogenic transformation of single mammary stem cell or due to transformation of mammary stem cells at various levels. ${ }^{9}$ CSCs markers are used to quantify the percentage of these cells in a cancer patient, with high values indicating a poor prognosis.

Understanding the histological, molecular and functional characteristics of a tumor will be necessary as tools for individualized/personalized cancer therapy in the future. ${ }^{2}$ Ambiguities in $B C$ classification still exist, with clinical routine still relying primarily on ER, PR and HER2 immunohistochemistry as a basis for classification while numerous studies have been carried out on molecular characterization of $\mathrm{BC}$.

Protein expression levels in $\mathrm{BC}$ and their role in classification has not been thoroughly investigated. Recently mass spectrometry (MS) based proteomic studies have been carried out with this objective..$^{10,11}$ In the Clinical Proteomic Tumor Analysis Consortium (CPTAC) study, ${ }^{11}$ novel proteomic and phosphoproteomic 
subgroups were identified through an unsupervised tumor classification. The three proteomic groups identified included one corresponding to the luminal cluster, second to the basal BC cluster and the third was named as stromal-enriched cluster composed of tumors from different subtypes. ${ }^{6}$

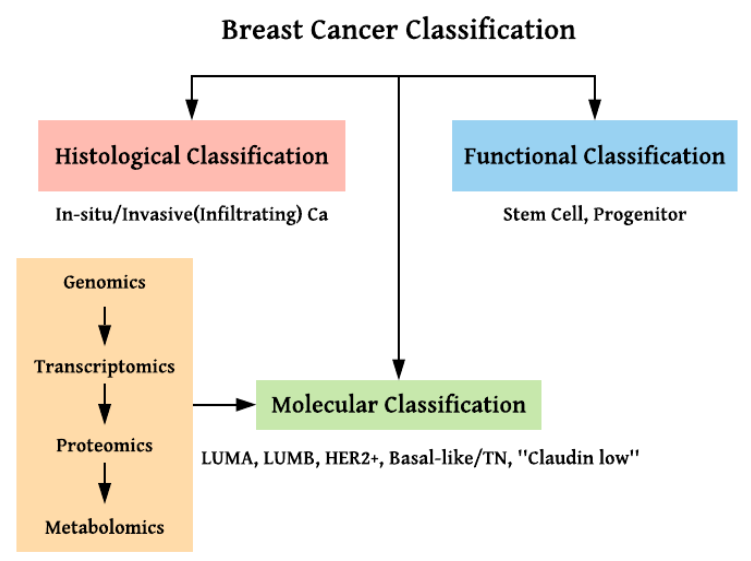

Figure 1: Evolution of breast cancer classification.

LUMA/LUMB: luminal A/B, TN: triple negative, Ca: cancer

Revere-Phase Liquid Chromatography-Mass Spectrometry (UHPLC - Thermo Fisher Scientific) was used for separation of peptides. Raw MS data was analyzed by MaxQuant (version 1.5.3.16) ${ }^{12}$ and Andomeda (an integrated peptide search engine). ${ }^{13}$ Hierarchical clustering analysis was carried out using $R$ environment (version 3.2.3) and ConsensusClusterPlus package. ${ }^{14}$ For information regarding protein-protein interaction in each group, the statistically significant upregulated proteins in each cluster were applied to the String database (http://string-db.org). This was followed by centrality analysis (identification of central nodes i.e. nodes appearing in shortest path between two other nodes) ${ }^{6}$ employing/with CytoNCA plug-in and Cytoscope..$^{15}$ The Cancer Proteome Atlas (TCPA) provided The Cancer Genome Atlas Reverse Phase Protein Array (TCGA RPPA) data.

The study by Yanovich and colleagues for proteomic classification of $B C$ has identified a novel luminal $A$ subtype with an intrinsic ER positive signature along with additional high expression of key signaling markers specific for TNBC. ${ }^{6}$ This finding suggests use of protein expression pattern for classification of luminal breast cancers, which might benefit treatment decision making.
Cancer proteomics is a robust high-throughput 'omic' technology, which can be used independently and also complementary to other 'omics' like genomics, transcriptomics and metabolomics.

Metabolomics is an "omics" science, a survey to identify and quantify low molecular weight metabolites that reflect alterations in energy metabolism. They are present downstream and are a reflection of the activity of genes, proteins and mRNA upstream. High throughput analytical techniques like mass spectrometry (MS) or nuclear magnetic resonance (NMR) are used for metabolic characterization using untargeted or targeted approaches. ${ }^{16}$ Metabolic profile can be obtained by analyzing the enormous raw data through specialized software with validation of the mass spectra obtained, with those available in the National Institute of Standards and Technology (NIST) Mass Spectral (Wiley registry) and Agilent Fiehn Metabolomics Retention Time Locked (RTL) libraries. A number of studies aimed at identifying a metabolomic biomarker for breast cancer have been carried out, ${ }^{16-18}$ however validation studies combining different sample types (e.g. blood, tissue, urine etc.) might bring an understanding of the overall metabolome of $\mathrm{BC}$. Although cancer metabolomics has evolved over time, it is still lagging behind in clinical diagnostics. ${ }^{19}$

The integration of multiple 'omics', including genomics, transcriptomics, proteomics and metabolomics will provide information regarding tumor progression, distant spread, and response to therapy. These experiments can yield high-accuracy and more robust data, exploring the complex heterogeneity of $\mathrm{BC}$, highlighting the importance of an in-depth multi-level point of view in BC research. This will also ensure tailored healthcare involving disease prevention, diagnosis, and treatment in this soon-to-come era of personalized medicine

\section{References}

1. Stingl J, Caldas C. Molecular heterogeneity of breast carcinomas and the cancer stem cell hypothesis. Nat Rev Cancer. 2007; 7: 791-9. doi:10.1038/nrc2212. 
2. Malhotra GK, Zhao X, Band H, Band V. Histological, molecular and functional subtypes of breast cancers. Cancer Biol Ther. 2010; 10: 955-60. doi:10.4161/cbt.10.10.13879

3. Sorlie T, Perou CM, Tibshirani R, Aas T, Geisler S, Johnsen $\mathrm{H}$, et al. Gene expression patterns of breast carcinomas distinguish tumor subclasses with clinical implications. Proc Natl Acad Sci USA. 2001; 98: 10869-74.

4. Sorlie T, Tibshirani R, Parker J, Hastie T, Marron JS, Nobel A, et al. Repeated observation of breast tumor subtypes in independent gene expression data sets. Proc Natl Acad Sci USA. 2003; 100: 8418-23.

5. Prat A, Parker JS, Karginova O, Fan C, Livasy C, Herschkowitz Jl, et al. Phenotypic and molecular characterization of the claudin-low intrinsic subtype of breast cancer. Breast Cancer Res. 2010; 12: 68.

6. Yanovich G, Agmo H, Harel M, Sonnenblick A, Peretz T, Geiger T. Clinical Proteomics of Breast Cancer Reveals a Novel Layer of Breast Cancer Classification. Cancer Res. 2018; 78(20): 6001-10. doi: 10.1158/0008-5472.CAN-18-1079.

7. Parker JS, Mullins M, Cheang MC, Leung S, Voduc $D$, Vickery $T$, et al. Supervised risk predictor of breast cancer based on intrinsic subtypes. J Clin Oncol. 2009; 27: 1160-7.

8. Ross JS, Hatzis C, Symmans WF, Pusztai L, Hortobagyi GN. Commercialized multigene predictors of clinical outcome for breast cancer. Oncologist. 2008; 13: 477-93.

9. Visvader JE. Keeping abreast of the mammary epithelial hierarchy and breast tumorigenesis. Genes Dev. 2009; 3: 2563-77.

10. Tyanova S, Albrechtsen R, Kronqvist $P$, Cox J, Mann M, Geiger T. Proteomic maps of breast cancer subtypes. Nat Commun. 2016; 710259.

11. Mertins $P$, Mani DR, Ruggles KV, Gillette MA, Clauser KR, Wang $P$, et al. Proteogenomics connects somatic mutations to signalling in breast cancer. Nature. 2016; 534(7605): 55-62. [PubMed: 27251275]

12. Tyanova S, Temu T, Cox J. The MaxQuant computational platform for mass spectrometry-based shotgun proteomics. Nat Protoc. 2016; 11(12): 230119. [PubMed: 27809316]

13. Cox J, Neuhauser N, Michalski A, Scheltema RA, Olsen JV, Mann M. Andromeda: a peptide search engine integrated into the MaxQuant environment. J Proteome Res. 2011; 10(4): 1794-805. [PubMed: 21254760]

14. Wilkerson MD, Hayes DN. ConsensusClusterPlus: a class discovery tool with confidence assessments and item tracking. Bioinformatics. 2010; 26(12): 1572-3. [PubMed: 20427518]

15. Tang Y, Li M, Wang J, Pan Y, Wu FX. CytoNCA: a cytoscape plugin for centrality analysis and evaluation of protein interaction networks. Biosystems. 2015; 127: 67-72. [PubMed: 25451770]

16. Asiago VM, Alvarado LZ, Shanaiah N, Gowda GA, Owusu-Sarfo K, Ballas RA et al. Early detection of breast cancer using metabolite profiling. Cancer Res. 2010; 70 (21): 8309-18. doi: 10.1158/00085472.CAN-10-1319.

17. Kanaan YM, Sampey BP, Beyene D, Esnakula AK, Naab TJ, Ricks-Santi LJ, et al. Metabolic profile of triple-negative breast cancer in African American women reveals potential biomarkers of aggressive disease. Cancer Genomics and proteomics. 2014; 11(6): 279-94.

18. Armitage EG, Southam AD. Monitoring cancer prognosis, diagnosis and treatment efficacy using metabolomics and lipidomics. Metabolomics. 2016; 12: 146.

19. Gunther UL. Metabolomic biomarkers for breast cancer. Pthobiology. 2015; 82: 153-65. doi: 10.1159/000430844. 